\title{
Some Extensions of Generalized Morphic Rings and EM-rings
}

\author{
Manal Ghanem and Emad Abu Osba
}

\begin{abstract}
Let $\mathrm{R}$ be a commutative ring with unity. The main objective of this article is to study the relationships between PP-rings, generalized morphic rings and EM-rings. Although PP-rings are included in the later rings, the converse is not in general true. We put necessary and sufficient conditions to ensure the converse using idealization and polynomial rings
\end{abstract}

\section{Introduction}

All rings are assumed to be commutative with unity 1 . Let $Z(R)$ be the set of all zero divisors in $R$, and let $\operatorname{reg}(R)=R \backslash Z(R)$.

A ring $R$ is called a morphic ring if for each $a \in R$, there exists $b \in R$ such that $A n n(a)=b R$ and $A n n(b)=a R$. It is known that for reduced commutative rings, morphic rings are equivalent to von Neumann regular rings. A ring $R$ is called generalized morphic ring if $\operatorname{Ann}(a)$ is principal for each $a \in R$, for more details, see [10], [12], [13] and [14]. It is clear that the class of generalized morphic rings includes a wide range of rings such as integral domains, principal ideal rings, von Neumann regular rings, PP-rings, etc. If for each polynomial $f(x) \in Z(R[x])$ there exists $c_{f} \in R$ and $f_{1}(x) \in \operatorname{reg}(R[x])$ such that $f(x)=c_{f} f_{1}(x)$, then $R$ is called an EM-ring. Note that in this case $A n n_{R[x]}(f)=A n n_{R[x]}\left(c_{f}\right)$, which simplifies working and characterizing

Key Words: polynomial ring; annihilating content; EM-ring; generalized morphic ring; PP-ring

2010 Mathematics Subject Classification: 13F20, 13F25, 13 E05.

Received:31.01.2017

Accepted:23.05.2017 
zero-divisors in $R[x]$. These rings were defined and characterized in [2], and it was shown there that this class includes a wide range of rings.

It is shown in [2] that if $R$ is a Noetherian ring, then $R$ is generalized morphic if and only if it is an EM-ring. In fact the Noetherian condition is not necessary as will be shown later on.

Recall that if $R$ is a ring, and $M$ is an $R$-module, then the idealization $R(+) M$ is the set of all ordered pairs $(r, m) \in R \times M$, equipped with addition defined by $(r, m)+(s, n)=(r+s, m+n)$ and multiplication defined by $(r, m)(s, n)=(r s, r n+s m)$. It is well-known that $R(+) R \simeq R[x] /\left(x^{2}\right)$. For the general case, we consider the ring $R[x] /\left(x^{n+1}\right)$, where $n \in \mathbb{N}$. In this case we set $R[x] /\left(x^{n+1}\right)=\left\{\sum_{i=0}^{n} a_{i} X^{i}: a_{i} \in R, X=x+\left(x^{n+1}\right)\right\}$.

A ring $R$ is called a PP-ring if every principal ideal of $R$ is a projective $R$-module. It is well known that $R$ is a PP-ring if and only if for each $a \in R, \operatorname{Ann}(a)$ is generated by an idempotent. A ring $R$ is called a PF-ring if every principal ideal of $R$ is a flat $R$-module. It is well known that $R$ is a PF-ring if and only if for each $a \in R, \operatorname{Ann}(a)$ is pure, i.e. for each $b \in \operatorname{Ann}(a)$, there exists $c \in \operatorname{Ann}(a)$ such that $b=b c$.

It is clear that a PP-ring is generalized morphic ring, and it was shown in [2] that a PP-ring is also an EM-ring, while $\mathbb{Z}_{4}$ is generalized morphic EM-ring that is not PP-ring.

In this article we will characterize when some extensions of a generalized morphic ring are generalized morphic. To be more precise; we will characterize when the polynomial ring, the ring $R[x] /\left(x^{n+1}\right)$ and the idealization of a generalized morphic ring is generalized morphic. We show that the later two rings are generalized morphic if and only if their base ring $R$ is a PP-ring.

We will characterize when the idealization of an EM-ring is an EM-ring. We will also continue the investigation of the polynomial rings of EM-rings we started in [2].

The following two lemmas will be used frequently in the following work.

Lemma 1.1. Let $R$ be a reduced ring. If $(a, x),(b, y) \in R(+) R$ such that $(a, x)(b, y)=(0,0)$, then $a b=a y=b x=0$.

Proof. We have $(0,0)=(a b, a y+b x)$, and so,

$$
\begin{gathered}
a b=0, \\
a y+b x=0, \\
0=a(a y+b x)=a^{2} y+a b x=a^{2} y=0 .
\end{gathered}
$$


Thus, $(a y)^{2}=0$, and since $R$ is reduced we have $a y=0$, whence $b x=0$.

Lemma 1.2. Let $R$ be a ring, and let $S=\left\{\left(a_{1}, b_{1}\right), \ldots,\left(a_{n}, b_{n}\right)\right\} \subseteq R(+) R$. Then $\operatorname{Ann}(S) \neq\{(0,0)\}$ if and only if $\operatorname{Ann}\left(a_{1}, \ldots, a_{n}\right) \neq\{0\}$.

Proof. Assume that $(a, b) \neq(0,0)$ and $(a, b)\left(a_{i}, b_{i}\right)=(0,0)$ for all $i$. Then $a a_{i}=0$ for all $i$. If $a=0$, then $b \neq 0$ and $b a_{i}=0$ for all $i$. Thus $\operatorname{Ann}\left(a_{1}, \ldots, a_{n}\right) \neq\{0\}$.

Now, if $a \neq 0$ and $a a_{i}=0$ for all $i$, then $(0, a)\left(a_{i}, b_{i}\right)=(0,0)$ for all $i$. Thus $\operatorname{Ann}(S) \neq\{(0,0)\}$.

Recall that for any ring $R$, the set $\operatorname{Min}(R)$ is the set of all minimal prime ideals of $R$, equipped with the hull kernel topology, and for any set $I$ of $R, V(I)=\{P \in \operatorname{Min}(R): I \subseteq P\}$, and $\operatorname{Supp}(I)=V(\operatorname{Ann}(I))$. An ideal $I$ of $R$ is called a $z^{0}-i$ deal, if whenever $V(a) \subseteq V(b)$, with $a \in I$, we have $b \in I$.

\section{Generalized Morphic Rings}

In this section we will relate reduced generalized morphic rings to complemented rings, and characterize when the polynomial ring of a generalized morphic ring is generalized morphic, and characterize generalized morphic rings using their minimal prime ideals.

A ring $R$ is called complemented if for each $a \in R$, there exists $b \in R$ such that $a b=0$ and $a+b \in \operatorname{reg}(R)$. A reduced $\operatorname{ring} R$ is complemented if and only if for each $a \in R$ there exists $b \in R$ such that $\operatorname{Ann}(\operatorname{Ann}(a))=\operatorname{Ann}(b)$. For more properties of complemented reduced rings, see Theorem 2.2 and Proposition 2.5 in [5], and Theorem 4.5 in [9].

It is clear that if $R$ is a reduced generalized morphic ring, then for any $a \in R$, there exists $b \in R$ such that $A n n(a)=b R$, and so, $\operatorname{Ann}(\operatorname{Ann}(a))=$ $A n n(b)$. Thus $R$ is a complemented ring.

For a complemented ring that is not generalized morphic, see Example 5.8 in [7] together with Theorem 1.3 in [8] and Theorem 2.2 below.

Recall that a ring $R$ is said to be Armendariz if the product of two polynomials in $R[x]$ is zero if and only if the product of their coefficients is zero.

We now characterize the case at which the polynomial ring of a generalized morphic ring is generalized morphic.

Theorem 2.1. If $R[x]$ is a generalized morphic ring, then $R$ is generalized morphic. If $R$ is Armendariz, then the converse is also true.

Proof. Assume $R[x]$ is generalized morphic, and let $a \in Z^{*}(R)$. Then $A n n_{R[x]}(a)=$

$f(x) R[x]$, where $f(x)=\sum_{i=0}^{n} a_{i} x^{i}$. Let $j$ be the least index such that $a_{j} \neq 0$. 
Then $a a_{j}=0$, and so, $a_{j} R \subseteq A n n_{R}(a)$. Let $b \in A n n_{R}(a) \backslash\{0\}$. Then $b=f(x) g(x)$ for some $g(x)=\sum_{i=0}^{m} b_{i} x^{i} \in R[x]$. Then $b=a_{0} b_{0} \in a_{0} R$, and $a_{0} \neq 0$. Thus $j=0$ and $A n n_{R}(a)=a_{0} R$ is principal, and hence $R$ is generalized morphic.

For the converse assume $R$ is Armendariz generalized morphic and let $f(x)=\sum_{i=0}^{n} a_{i} x^{i} \in Z(R[x])$. Then there exists $a \in R$ such that $a a_{i}=0$ for all $i$. Thus $\{0\} \neq A n n_{R}\left(a_{0}, a_{1}, \ldots, a_{n}\right)$. Since $R$ is generalized morphic, there exists $b \in R$ such that $A n n_{R}\left(a_{0}, a_{1}, \ldots, a_{n}\right)=b R$, see Theorem 5 in [12]. Thus $b R[x] \subseteq A n n_{R[x]}(f)$. If $g(x)=\sum_{i=0}^{m} c_{i} x^{i} \in A n n(f)$, then $c_{i} a_{j}=0$ for all $i$ and $j$, since $R$ is Armendariz, and so, $c_{i} \in b R$ for each $i$ and $g(x) \in b R[x]$. Thus $A n n_{R[x]}(f)=b R[x]$ is principal and $R[x]$ is generalized morphic.

Question: While there are non-commutative generalized morphic rings that are non-Armendariz, is it necessary for a commutative generalized morphic ring to be Armendariz?

Next, we will characterize generalized morphic reduced rings using minimal prime ideals, and the concept of $z^{0}$-ideals, borrowed from the rings of continuous functions.

Theorem 2.2. Let $R$ be a reduced ring. Then $R$ is a generalized morphic ring if and only if for each $a \in R$ there exists $b \in R$ such that $\operatorname{Supp}(a)=V(b)$ and $b R$ is a $z^{0}$-ideal.

Proof. Assume $R$ is a generalized morphic ring, and let $a \in R$. Then $\operatorname{Ann}(a)=$ $b R$ for some $b \in R$. So we have $\operatorname{Supp}(a)=V(\operatorname{Ann}(a))=V(b R)=V(b)$. Moreover, if $V(b r) \subseteq V(c)$, then $V(b) \subseteq V(b r) \subseteq V(c)$, and so, for each $P \in \operatorname{Min}(R)$, if $b \in P$, then $c \in P$ and hence, $a c \in P$. If $b \notin P$, then $a \in A n n(b) \subseteq P$, and so, $a c \in P$. Thus, $a c \in \bigcap_{P \in M i n(R)} P=\{0\}$, since $R$ is reduced. Therefore, $c \in \operatorname{Ann}(a)=b R$, and $b R$ is a $z^{0}$-ideal.

Conversely, assume $a, b \in R$ such that $\operatorname{Supp}(a)=V(b)$ and $b R$ is a $z^{0}$-ideal. Let $P \in \operatorname{Min}(R)$. If $a \in P$, then $a b \in P$. If $a \notin P$, then $\operatorname{Ann}(a) \subseteq$ $P$, and so, $P \in \operatorname{Supp}(a)=V(b)$. Hence, $b \in P$, and so, $a b \in P$. Thus, $a b \in \bigcap_{P \in \operatorname{Min}(R)} P=\{0\}$, which implies that $b R \subseteq \operatorname{Ann}(a)$. If $c \in \operatorname{Ann}(a)$, then we have $V(b)=\operatorname{Supp}(a) \subseteq V(c)$, and so, $c \in b R$, being a $z^{0}$-ideal. Hence $A n n(a)=b R$, and $R$ is a generalized morphic ring. 


\section{When is $R[x] /\left(x^{n+1}\right)$ Generalized Morphic ring?}

In this section we characterize the case at which the idealization of a generalized morphic ring or more generally, the ring $R[x] /\left(x^{n+1}\right)$ is generalized morphic.

Theorem 3.1. Let $R$ be a ring, $M$ an $R$-module and let $S=R(+) M$. If $S$ is generalized morphic ring, then $R$ is generalized morphic ring.

Proof. Let $a \in Z\left(R^{*}\right)$. Then $A n n((a, 0))=(r, m) S$, and hence $(0,0)=$ $(a, 0)(r, m)=(a r, a m)$. So, $a r=0$, and thus, $r R \subseteq A n n(a)$. Now, if $x \in \operatorname{Ann}(a)$, then $(x, 0)(a, 0)=(x a, 0)=(0,0)$.

But in this case, we must have $(x, 0)=(r, m)(t, s)=(r t, r s+t m)$, for some $(t, s) \in S$. So, $x \in r R$. Therefore, $\operatorname{Ann}(a)=r R$, and hence, $R$ is generalized morphic ring.

The converse of the above Theorem needs not be true, since $\mathbb{Z}_{4}$ is a generalized morphic ring, while $\mathbb{Z}_{4}(+) \mathbb{Z}_{4}$ is not.

Now, the question is, for what rings $R$, the converse of this Theorem must be true. In the following, we will give the answer. But first we recall the following proposition which was proved in [12].

Proposition 3.2. Let $R$ be a reduced ring. Then the following are equivalent:

(1) The ring $R$ is morphic.

(2) The ring $R[x] /\left(x^{n+1}\right)$ is morphic for each $n \in \mathbb{N}$.

(3) The ring $R(+) R$ is morphic.

(4) The ring $R$ is von Neumann regular ring.

In the following, we will prove an analogue result for the equivalence of PP-rings and generalized morphic idealization.

Lemma 3.3. Let $R$ be a reduced ring, and let $f=\sum_{i=0}^{n} a_{i} X^{i} \in Z\left(R[x] /\left(x^{n+1}\right)\right) \backslash$ $\{0\}, g=\sum_{i=0}^{n} b_{i} X^{i}=\operatorname{Ann}(f)$. Then $b_{i} \in \operatorname{Ann}\left(a_{0}, a_{1}, \ldots, a_{n-i}\right)$ for $i=$ $0,1,2, \ldots, n$.

Proof. Since $f g=0$, we have $\sum_{i=0}^{j} a_{i} b_{j-i}=0$, for $j=0,1,2, \ldots, n$. Thus, $a_{0} b_{0}=0$, and if $b_{0} \in \operatorname{Ann}\left(a_{0}, a_{1}, \ldots, a_{j}\right), j<n$, then multiplying the equation $a_{0} b_{j+1}+a_{1} b_{j}+\ldots+a_{j} b_{1}+a_{j+1} b_{0}=0$ by $b_{0}$ yields $a_{j+1} b_{0}^{2}=0$, and since $R$ is reduced we have $a_{j+1} b_{0}=0$, i.e. $b_{0} \in \operatorname{Ann}\left(a_{0}, a_{1}, \ldots, a_{j+1}\right)$. Hence $b_{0} \in \operatorname{Ann}\left(a_{0}, a_{1}, \ldots, a_{n}\right)$. Now, assume that $b_{i} \in \operatorname{Ann}\left(a_{0}, a_{1}, \ldots, a_{n-i}\right)$, for $i=0,1, \ldots, j<n$, then the equation $a_{0} b_{j+1}+a_{1} b_{j}+\ldots+a_{j} b_{1}+a_{j+1} b_{0}=0$ 
reduces to $a_{0} b_{j+1}=0$. So, assume that $a_{k} b_{j+1}=0$, for $k=0,1, \ldots, l<n-j-1$, then the equation $\sum_{s+k=l+1+j+1} a_{k} b_{s}=0$, reduces to $a_{l+1} b_{j+1}=0$, and so we have $b_{j+1} \in \operatorname{Ann}\left(a_{0}, a_{1}, \ldots, a_{n-j-1}\right)$.

Theorem 3.4. The following are equivalent for a ring $R$ :

(1) The ring $R$ is a PP-ring.

(2) The ring $R[x] /\left(x^{n+1}\right)$ is generalized morphic for each $n \in \mathbb{N}$.

(3) The ring $S=R(+) R$ is generalized morphic.

(4) The ring $R$ is generalized morphic PF-ring.

(5) The ring $R$ is complemented PF-ring.

Proof. (1) $\Rightarrow(2)$ Assume $R$ is a PP-ring, and $f=\sum_{i=0}^{n} a_{i} X^{i} \in Z\left(R[x] /\left(x^{n+1}\right)\right) \backslash$ $\{0\}, g=\sum_{i=0}^{n} b_{i} X^{i}=A n n(f)$. Then it follows by Lemma 3.3 that $b_{i} \in$ $\operatorname{Ann}\left(a_{0}, a_{1}, \ldots, a_{n-i}\right)=e_{i} R$, where $e_{i}^{2}=e_{i}$ for $i=0,1,2, \ldots, n$, and in this case we would have $e_{i} e_{j}=e_{i}$, whenever $i \leq j$. Let $e=\sum_{i=0}^{n} e_{i} X^{i}$. Then it is clear that $e \in A n n(f)$. Let $K_{0}=b_{0}-b_{0} X, K_{1}=b_{1}\left(1-e_{0}\right)-b_{1} X+2 b_{1} e_{0} X-b_{0} e_{0} X^{2}$. Then it is clear that $b_{i} X^{i}=e K_{i}$ for $i=0,1$.

Now, for $1<m \leq n$, let $T_{m}=b_{m}\left(1-e_{m-1}\right)-b_{m} X+2 b_{m} e_{m-1} X-$ $b_{m} e_{m-1} X^{2}$. Then routine computations yields that $e T_{m}=b_{m} X^{m}+\sum_{j=0}^{m-2} b_{m} e_{j} X^{j+1}-$ $\sum_{j=0}^{m-2} b_{m} e_{j} X^{j+2}$. Let $G_{m, i}=-b_{m} e_{m-1-i} X^{i}+2 b_{m} e_{m-1-i} X^{i+1}-b_{m} e_{m-1-i} X^{i+2}$, for all $1 \leq i \leq m-1$. Then $e G_{m, i}=-b_{m} e_{m-1-i} X^{m-1}+b_{m} e_{m-1-i} X^{m}-$ $\sum_{j=0}^{m-2-i} b_{m} e_{j} X^{j+i}+2 \sum_{j=0}^{m-2-i} b_{m} e_{j} X^{j+i+1}-\sum_{j=0}^{m-2-i} b_{m} e_{j} X^{j+i+2}$, for $1 \leq i \leq m-2$, and $e G_{m, m-1}=-b_{m} e_{0} X^{m-1}+b_{m} e_{0} X^{m}$. Let $k_{m, r}=T_{m}+\sum_{i=1}^{r} G_{m, i}$. Using finite induction, one can show that $e k_{m, r}=b_{m} X^{m}+\sum_{j=0}^{m-2-r} b_{m} e_{j} X^{j+r+1}-$ $\sum_{j=0}^{m-2-r} b_{m} e_{j} X^{j+r+2}$, for $1 \leq r \leq m-2$, and $e K_{m, m-2}=b_{m} X^{m}+b_{m} e_{0} X^{m-1}-$ $b_{m} e_{0} X^{m}$. Now, let $K_{m}=\left(T_{m}+\sum_{i=1}^{m-2} G_{m, i}+G_{m, m-1}\right)$, for $1<m \leq n$. Then $e K_{m}=b_{m} X^{m}$, and so, $g=e \sum_{m=0}^{n} K_{m}$. Thus, $A n n(f)=(e)$, and $R[x] /\left(x^{n+1}\right)$ is generalized morphic.

$(2) \Rightarrow(3)$ Clear, since $R(+) R$ is isomorphic to $R[x] /\left(x^{2}\right)$. 
$(3) \Rightarrow(1)$ Assume that $S$ is generalized morphic, and let $a \in Z(R) \backslash\{0\}$. Then $(0, a) \in Z(S) \backslash\{(0,0)\}$, and so, $\operatorname{Ann}(0, a)=(x, y) S$. It is clear that $x R \subseteq \operatorname{Ann}(a)$, and if $b \in \operatorname{Ann}(a)$, then $(b, 0)(0, a)=(0,0)$, and hence, $(b, 0)=$ $(x, y)(z, w)$. Thus $b=x z \in x R$, and therefore $\operatorname{Ann}(a)=x R$. But $(0,1)(0, a)=$ $(0,0)$, and so, $(0,1)=(x, y)(\alpha, \beta)$. Thus we have:

$$
\begin{gathered}
0=x \alpha, \\
1=x \beta+y \alpha,
\end{gathered}
$$

which yields that

$$
x=x^{2} \beta,
$$

and hence, $x \beta=(x \beta)^{2}$, and $\operatorname{Ann}(a)=(x \beta) R$. Thus $R$ is a PP-ring.

$(1) \Leftrightarrow(4)$ See Corollary 3.12 in [14].

$(1) \Leftrightarrow(5)$ See Proposition 2.7 in [11].

Example 3.5. Let $F$ be a field. Then $R=F[x, y] /(x y)$ is a reduced complemented ring that is not a PP-ring, see Remark 2 in [3], and Theorem 4.5 in [9]. One can see easily that $R$ is a generalized morphic ring, while $R[x] /\left(x^{n+1}\right)$ is not for any $n \in \mathbb{N}$.

It is immediate that if $R$ is a PF-ring that is not a PP-ring, then $R$ and $R(+) M$ are not generalized morphic for any $R$-module $M$.

Since PP-rings are always reduced, we conclude the following easily.

Corollary 3.6. If $R[x] /\left(x^{n+1}\right)$ is generalized morphic, then $R$ is reduced.

\section{Polynomial rings of EM-rings}

In [1], the concept of the annihilating content of a polynomial $f(x)$ was introduced to be a constant $c_{f}$ such that $f(x)=c_{f} f_{1}(x)$ with $f_{1}(x)$ is not a zero-divisor, and in [2], we called a ring $R$ to be an EM-ring if every zerodivisor polynomial in $R[x]$ has an annihilating content. Many properties of this ring were investigated, and many open problems were posed. We now study the polynomial ring of an EM-ring.

Theorem 4.1. If $R$ is an EM-ring, then $R[x]$ is an EM-ring. If $R$ is a reduced, then the converse is also true. 
Proof. Assume $R$ is an EM-ring. To show that $R[x]$ is an EM-ring, we will follow the proof of the result in the unpublished article [2]. Let $f(x, y)=$ $\sum_{i=0}^{n} f_{i}(x) y^{i}$ be zero-divisor in $R[x, y]=(R[x])[y]$. Then there exists nonzero $h(x)$ such that $h f_{i}=0$ for all $i$. Define

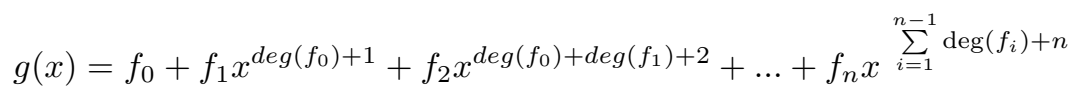

Since $h g=0$, there exists $c_{g} \in Z(R)$ and nonzero-divisor $g_{1}=\sum_{i=1}^{m} b_{i} x^{i}$ such that $g=c_{g} g_{1}$. So, $\cap A n n\left(b_{i}\right)=\{0\}$, and $f_{0}=c_{g} \sum_{i=0}^{\operatorname{deg}\left(f_{0}\right)} b_{i} x^{i}=c_{g} h_{0}(x), f_{1}=$ $c_{g} \sum_{i=0}^{\operatorname{deg}\left(f_{1}\right)} b_{i+\operatorname{deg}\left(f_{0}\right)+1} x^{i}=c_{g} h_{1}$, and so on. Hence, $f(x, y)=c_{g} \sum_{i=0}^{n} h_{i}(x) y^{i}$. If $\sum_{i=0}^{n} h_{i}(x) y^{i}$ is a zero-divisor, then there exists nonzero $k(x)$ such that $k(x) h_{i}(x)=$ 0 for each $i$. Define

$$
l(x)=\sum_{i=0}^{n} h_{i}(x) x^{\sum_{j<i} \operatorname{deg}\left(f_{j}\right)+1}
$$

and so, $k(x) l(x)=0$, and therefore there exists a nonzero $c \in R$ such that $c h_{i}(x)=0$, and so, $c b_{i}=0$ for all $i$, a contradiction, since $\cap A n n\left(b_{i}\right)=$ $\{0\}$.Thus $\sum_{i=0}^{n} h_{i}(x) y^{i}$ is nonzero-divisor, and $R[x]$ is an EM-ring.

Assume now that $R$ is a reduced ring, and $R[x]$ is an EM-ring. Let $f(x)=$ $\sum_{i=0}^{l} a_{i} x^{i} \in Z(R[x]) \backslash\{0\}$. Then $g(y)=\sum_{i=0}^{l} a_{i} y^{i} \in Z((R[x])[y]) \backslash\{0\}$, and so, there exists $h(x)=\sum_{i=0}^{m} h_{i} x^{i} \in R[x]$ such that $g(y)=h(x) \sum_{i=0}^{l} k_{i}(x) y^{i}$, with $\bigcap \operatorname{Ann}\left(k_{i}(x)\right)=\{0\}$. Assume that $k_{i}(x)=\sum_{j=0}^{n_{i}} k_{i, j} x^{j}$, which implies that $\bigcap A n n\left(k_{i, j}\right)=\{0\}$. Note that $a_{i}=h(x) k_{i}(x)=h_{0} k_{0}$. But $h(x) k_{i}(x)=$ $\sum_{k=0}^{m+n_{i}} c_{k} x^{k}$, with $c_{k}=\sum_{j=0}^{k} h_{j} k_{i, k-j}$. Now we have:

$$
\begin{gathered}
0=c_{m+n_{i}}=h_{m} k_{i, n_{i}} \\
0=c_{m+n_{i}-1}=h_{m} k_{i, n_{i}-1}+h_{m-1} k_{i, n_{i}},
\end{gathered}
$$

which implies that $0=h_{m}^{2} k_{i, n_{i}-1}$, and so, $0=h_{m} k_{i, n_{i}-1}$, since $R$ is reduced.

$$
0=c_{m+n_{i}-2}=h_{m} k_{i, n_{i}-2}+h_{m-1} k_{i, n_{i}-1}+h_{m-1} k_{i, n_{i}},
$$


which implies that $0=h_{m}^{2} k_{i, n_{i}-2}$, and so, $0=h_{m} k_{i, n_{i}-2}$

Now, assume we have $h_{m} k_{i, s}=0$, for $s=n_{i}, n_{i}-1, \ldots, j+1$. Thus we have

$$
0=c_{m+j}=h_{m} k_{i, j}+h_{m-1} k_{i, j+1}+\ldots h_{j} k_{i, m},
$$

which implies that $0=h_{m}^{2} k_{i, j}$, and so, $0=h_{m} k_{i, j}$, this shows that $h_{m} k_{i, s}=$ 0 , for $s=0,1,2, \ldots, n_{i}$.

Thus, $h(x) k_{i}(x)=\left(h(x)-h_{m} x^{m}\right) k_{i}(x)$.

Continue to get $h(x)=h_{0} k_{i}(x)$, which implies that $h_{0} k_{i, j}=0$ for all $j \in\left\{1,2, \ldots, n_{j}\right\}$, and $i \in\{1,2, \ldots, l\}$

Now define $w(x)=\sum_{i=0}^{n} k_{i, 0} x^{i}+x^{n_{0}+1} \sum_{j=1}^{n_{0}} k_{0, j} x^{j}+x^{n_{0}+n_{1}+2} \sum_{j=1}^{n_{1}} k_{1, j} x^{j}+\ldots+$ $x^{n_{0}+n_{1}+\ldots+n_{l-1}+l} \sum_{j=1}^{n_{l}} k_{l, j} x^{j}$. Then $\operatorname{Ann}(w)=\{0\}$, and $f(x)=h_{0} w(x)$. Hence, $R$ is an EM-ring.

Question: Is the above result true for nonreduced rings?

\section{Idealization of EM-rings}

It was shown in [2] that if $R$ is a Noetherian ring, then $R$ is an EM-ring if and only if it is a generalized morphic ring, and an example was given for an EM-ring that is not generalized morphic, but the precise relation between the two concepts was not accomplished. In the following, we will give a partial answer.

We now investigate the idealization of EM-rings, and relate it to generalized morphic rings.

Theorem 5.1. Assume $R$ is a ring such that $S=R(+) R$ is an EM-ring, then $R$ is an EM-ring.

Proof. Let $f(x)=\sum_{i=0}^{n} a_{i} x^{i} \in Z(R[x]) \backslash\{0\}$. Then there exists $a \in R \backslash\{0\}$ such that $a a_{i}=0$ for each $i$. Let $g(x)=\sum_{i=0}^{n}\left(a_{i}, 0\right) x^{i} \in S[x]$. Then $(a, 0)\left(a_{i}, 0\right)=$ $(0,0)$ for each $i$, and so, $g(x) \in Z(S[x]) \backslash\{(0,0)\}$. Thus there exists $(r, m) \in S$ such that $g(x)=(r, m) \sum_{i=0}^{k}\left(r_{i}, m_{i}\right) x^{i}$, with $\bigcap_{i=0}^{k} A n n\left(r_{i}, m_{i}\right)=\{(0,0)\}, n \leq k$. Hence, we have $\bigcap_{i=0}^{k} A n n\left(r_{i}\right)=\{0\}$, and $f(x)=r \sum_{i=0}^{k} r_{i} x^{i}$. Thus, $R$ is an EM-ring. 
The converse of the above Theorem needs not be true, since $\mathbb{Z}_{4}$ is an EMring, while $\mathbb{Z}_{4}(+) \mathbb{Z}_{4}$ is not.

In [2], we showed that if $R$ is a PP-ring, then it is an EM-ring. We now give a more precise result.

Theorem 5.2. A ring $R$ is a PP-ring if and only if $S=R(+) R$ is an EMring.

Proof. Assume that $R$ is a PP-ring, and $f(x)=\sum_{i=0}^{n}\left(a_{i}, b_{i}\right) x^{i} \in Z(S[x]) \backslash$ $\{(0,0)\}$. Since $R$ is a PP-ring, we can write $a_{i}=u_{i} r_{i}$, and $b_{i}=v_{i} s_{i}$, where $u_{i}$ and $v_{i}$ are idempotents, $r_{i}$ and $s_{i}$ are regular elements for each $i$, see [4, Lemma 2]. Define the idempotents $u, v$ and $e$ as follows:

$$
\begin{gathered}
1-u=\prod_{i=0}^{n}\left(1-u_{i}\right), \\
1-v=\prod_{i=0}^{n}\left(1-v_{i}\right), \\
1-e=(1-u)(1-v) .
\end{gathered}
$$

Note that $\left(a_{i}, 0\right)=(u, e-u)\left(a_{i}, 0\right)$ and $\left(0, b_{i}\right)=(u, e-u)\left((1-u)\left(b_{i}+1-\right.\right.$ $\left.e), b_{i}\right)$, and so, $\sum_{i=0}^{n}\left(a_{i}, b_{i}\right) x^{i}=(u, e-u) \sum_{i=0}^{n}\left(a_{i}+(1-u)\left(b_{i}+1-e\right), b_{i}\right) x^{i}$. Now, let $I$ be the ideal in $R$ generated by the elements $a_{i}+(1-u)\left(b_{i}+1-e\right)$. Then $a_{i}=u_{i}\left(a_{i}+(1-u)\left(b_{i}+1-e\right)\right) \in I$ for each $i$. Also, $(1-u)\left(b_{i}+1-e\right)=$ $a_{i}+(1-u)\left(b_{i}+1-e\right)-a_{i} \in I$ for each $i$, which implies that $(1-u) b_{i}=$ $e(1-u)\left(b_{i}+1-e\right) \in I$, since $e b_{i}=b_{i}$ for each $i$. Therefore, we have $1-e=$ $(1-e)(1-u) \in I$. Now, if $\alpha \in \operatorname{Ann}(I)$, then $0=\alpha a_{i}=\alpha u_{i} r_{i}$, and so, $\alpha u_{i}=0$ for each $i$, which implies that $\alpha u=0$, and so, $0=\alpha(1-u) b_{i}=\alpha b_{i}$ for each $i$. Thus, $\alpha v_{i}=0$ for each $i$. Hence we have $\alpha u=0=\alpha v$, and so, $\alpha e=0$. But we have also $\alpha(1-e)=0$, which implies that $\alpha=0$, i.e. $\operatorname{Ann}(I)=\{0\}$, and so it follows by Lemma 1.2 that $\sum_{i=0}^{n}\left(a_{i}+(1-u)\left(b_{i}+1-e\right), b_{i}\right) x^{i} \in \operatorname{reg}(S[x])$. Thus $S$ is an EM-ring.

Now assume that $S$ is an EM-ring, $b \in Z(R) \backslash\{0\}$ and let $a \in A n n(b) \backslash\{0\}$. Then $f(x)=(0,1)+(b, 0) x \in Z(S[x]) \backslash\{(0,0)\}$, since it is annihilated by $(0, a)$. Thus $f(x)=(\alpha, \beta) \sum_{i=0}^{n}\left(n_{i}, m_{i}\right) x^{i}$, with $\bigcap_{i} \operatorname{Ann}\left(n_{i}\right)=\{0\}$. Thus, we have:

$$
0=\alpha n_{0}
$$




$$
\begin{gathered}
1=\alpha m_{0}+\beta n_{0}, \\
b=\alpha n_{1}, \\
0=\alpha n_{i} \text { for all } i>1 .
\end{gathered}
$$

But $b=b\left(\alpha m_{0}+\beta n_{0}\right)=b \alpha m_{0}+\alpha n_{1} \beta n_{0}=b\left(\alpha m_{0}\right)$. Also note that $\alpha m_{0}=\left(\alpha m_{0}\right)^{2}+\alpha m_{0} \beta n_{0}=\left(\alpha m_{0}\right)^{2}$. Thus, Ann $\left(\alpha m_{0}\right) \subseteq$ Ann $(b)$. Now let $d \in \operatorname{Ann}(b)$. Then we have:

$$
\begin{gathered}
0=\left(d m_{0}\right) 0=\left(d m_{0}\right) \alpha n_{0}=\left(d \alpha m_{0}\right) n_{0}, \\
0=\left(d m_{0}\right) b=\left(d m_{0}\right) \alpha n_{1}=\left(d \alpha m_{0}\right) n_{1}, \\
0=\left(d m_{0}\right) 0=\left(d m_{0}\right) \alpha n_{i}=\left(d \alpha m_{0}\right) n_{i} \text { for all } i>1,
\end{gathered}
$$

which implies that $d \alpha m_{0} \in \bigcap_{i} A n n\left(n_{i}\right)=\{0\}$. Hence, $\operatorname{Ann}(b)=\operatorname{Ann}\left(\alpha m_{0}\right)=$ $\left(1-\alpha m_{0}\right) R$ is generated by an idempotent, and so, $R$ is a PP-ring.

Using Theorems 3.4 and 5.2, one can deduce the following:

Corollary 5.3. For any ring $R$, we have $R(+) R$ is an EM-ring if and only if it is generalized morphic.

Example 5.4. The space $X=\beta \mathbb{N} \backslash \mathbb{N}$ is an F-space that is not a basically disconnected space nor complemented, see [6, 6W and 14.27], and so, $C(X)$ is a reduced Bézout ring that is not a PP-ring. Thus $C(X)(+) C(X)$ is not an EM-ring. Also we have $C(X)$ is an EM-ring which is not generalized morphic.

Questions: It is still an open problem to characterize the relation between EM-rings and generalized morphic rings. Although they are not equivalent, we saw that $R(+) R$ is an EM-ring if and only if it is generalized morphic, even if $R$ was not Noetherian. We also don't know yet what sufficient conditions must be add to an EM-ring to become a PP-ring. It is not difficult to show that if $R[x] /\left(x^{n+1}\right)$ is an EM-ring, then $R$ is a PP-ring. We are still working for the other direction. 


\section{References}

[1] Abu Osba, E. and Alkam, O., When zero-divisor graphs are divisor graphs, Turk. J. Math, 41 (2017), 797- 807.

[2] Abu Osba, E. and Ghanem, M., Annihilating content in polynomial and power series rings, (Submitted).

[3] Artico, G. and Marconi, U., On the compactness of minimal spectrum. Rendiconti del Seminario Matematico della Università di Padova 56 (1976), 79-84.

[4] Endo, S., Note on P.P.rings, Nagoya Math J. 17 (1960), 167-170.

[5] Evans, M.W., On commutative P.P. rings, Pacific journal of mathematics 41(3) (1972), 687-697.

[6] Gillman, L. and Jerison, M., Rings of continuous functions, Graduate Texts in Math, 43, Berlin-Heidelberg, New York, 1976.

[7] Henriksen, M. and Jerison, M.,The space of minimal prime ideals of a commutative ring, Trans. Amer. Math. Soc. 115 (1965), 110-130.

[8] Henriksen, M. and Woods, R.G., Cozero complemented spaces; when the space of minimal prime ideals of a $\mathrm{C}(\mathrm{X})$ is compact, Topology and its Applications 141 (2004), 147-170.

[9] Huckaba, J., Commutative rings with zero divisors, Marcel Dekker, INC, New York, 1988.

[10] Liu, Q. and Chen, J., Coherence and generalized morphic property of triangular matrix rings, Communications in Algebra 42 (2014), 27882799 .

[11] Matlis, E., The minimal prime spectrum of a reduced ring, Illinois Journal of Mathematics 27(3) (1983), 353-391.

[12] Yang, X., On rings whose finitely generated left ideals are left annihilators of an element, arXiv:1002.3193

[13] Yuxian, G., The P-cotorsion dimensions of modules and rings, Acta Mathematica Scientia 30B(4) (2010), 1029-1043.

[14] Zhua, H. and Dinga, N., Generalized morphic rings and their applications, Communications in Algebra 35(7) (2007), 2820-2837. 
Manal GHANEM,

Department of Mathematics, School of Science,

The University of Jordan,

Amman 11942, Jordan,

Email: m.ghanem@ju.edu.jo

Emad ABU OSBA

Department of Mathematics, School of Science,

The University of Jordan,

Amman 11942, Jordan,

Email:eabuosba@ju.edu.jo 